HETEROCYCLES, Vol. 104, No. 1, 2022, pp. 85 - 93. @ 2022 The Japan Institute of Heterocyclic Chemistry Received, 14th September, 2021, Accepted, 15th October, 2021, Published online, 20th October, 2021 DOI: $10.3987 / C O M-21-14553$

\title{
SYNTHESIS AND REACTIVITY OF NOVEL 5-IMINO-1,2-OXAZOLE
}

\section{Khalid Widyan*}

Department of Chemistry, Tafila Technical University, P.O. Box 179, Tafila, Jordan, E-mail: k.widyan@ttu.edu.jo

\begin{abstract}
A convenient synthetic approach towards 5-imino-1,2-oxazoles beginning from $N$-substituted $C$-benzotriazolated nitrones and Reformatsky reagent is developed. The conversion of trisubstituted 5-imino-1,2-oxazoles into the corresponding 5-functionalized 1,2-oxazoles by interactions with phenyl isocyanates and $20 \% \mathrm{HCl}$ is provided. In addition, one-pot synthesis of 1,2-oxazole-5-ones is achieved with yield of $85-90 \%$.
\end{abstract}

\section{INTRODUCTION}

Development of simple methods for the synthesis of new analogs of bioactive heterocyclic compounds is of extreme importance in heterocyclic and medicinal chemistry. The yet unknown 5-imino-1,2-oxazoles (I) are analogs of 1,2-oxazol-5-ones (II) (Figure 1), a class of compounds which are useful synthetic intermediates for the preparation of heterocyclic compounds, ${ }^{1-4}$ and has attracted considerable attention in medicinal chemistry due to their pharmacological activity, 5,6 as well as remarkable activity as anti-inflammatory agents, ${ }^{5,7}$ and anti-cancer agents. ${ }^{8,9}$ They are also used for the treatment of central nervous system disorder. ${ }^{10,11}$
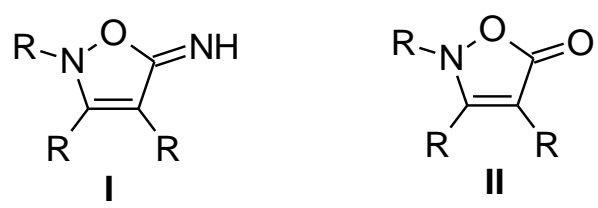

Figure 1. 5-Imino-1,2-oxazoles (I) and 1,2-oxazol-5-ones (II)

As part of our ongoing studies on applications of benzotriazole $(\mathrm{BtH})$ in organic synthesis and on development of new protocols for the synthesis of heterocyclic compounds we investigated the applicability of $C$-benzotriazolated nitrones for the synthesis of 5-imino-1,2-oxazoles $\mathbf{I}^{12-15}$ 


\section{RESULTS AND DISCUSSION}

Diaryl (heteroaryl) nitrones 1a-h were prepared following literature procedures. ${ }^{16-18}$ Treatment of 1a-h with chlorobenzotriazole $(\mathrm{BtCl})$ and sodium benzotriazolate $(\mathrm{BtNa})$ in THF furnished exclusively $\mathbf{2 a - h}$ in high overall yields according to an established procedure (Scheme 1). ${ }^{15}$

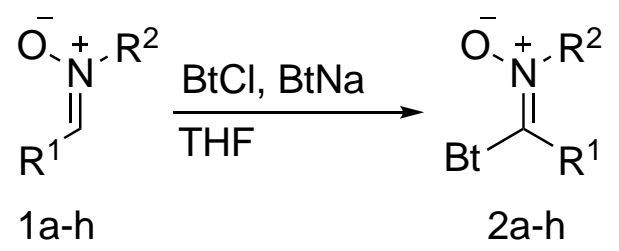

Scheme 1. Synthesis of $C$-benzotriazolated nitrones

Then, $N$-[(1H-benzotriazol-1-yl)-(4-methylphenyl)methylene]-4-methylbenzenamine oxide (2a) was used as the substrate to investigate the Reformatsky reaction of 2-bromopropionitrile (3a) to prepare 5-imino-4-methyl-2,3-di(4-methylphenyl)-2H-1,2-oxazole (4a) (Scheme 2).

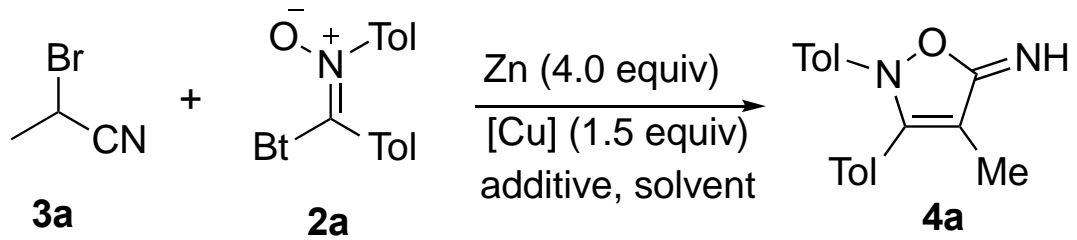

Scheme 2. Synthesis of 5-imino-1,2-oxazole 4a

Table 1. Screening of reaction conditions for synthesis of 5-imino-1,2-oxazole $\mathbf{4 a}$

\begin{tabular}{ccccccc}
\hline Entry & {$[\mathrm{Cu}]$} & Additive & Solvent & Temperature, ${ }^{\circ} \mathrm{C}$ & Time, $\mathrm{h}$ & Yield, $\%$ \\
\hline 1 & - & - & $\mathrm{THF}$ & $\mathrm{rt}$ & 5 & 20 \\
2 & - & - & $\mathrm{THF}$ & 60 & 5 & 35 \\
3 & - & - & $\mathrm{MeCN}$ & 60 & 3 & 38 \\
4 & $\mathrm{CuBr}$ & - & $\mathrm{MeCN}$ & 60 & 3 & 65 \\
5 & $\mathrm{CuI}$ & - & $\mathrm{MeCN}$ & 60 & 3 & 57 \\
6 & $\mathrm{CuBr}$ & $4 \AA \mathrm{MS}$ & $\mathrm{MeCN}$ & 60 & 3 & 85 \\
7 & $\mathrm{CuBr}$ & $4 \AA \mathrm{MS}$ & $\mathrm{THF}$ & 60 & 3 & 82 \\
8 & $\mathrm{CuBr}$ & $4 \AA \mathrm{MS}$ & $\mathrm{CH}_{2} \mathrm{Cl}$ & 40 & 3 & 15 \\
9 & $\mathrm{CuBr}$ & $4 \AA \mathrm{MS}$ & $\mathrm{PhMe}_{2}$ & 60 & 3 & 7 \\
\hline
\end{tabular}

When the reaction was carried out with 4.0 equivalents of zinc powder in THF at room temperature, the desired product $4 \mathbf{a}$ was obtained in $20 \%$ yield (Table1, entry 1). When the reaction was carried out at $60{ }^{\circ} \mathrm{C}$ in THF or MeCN, compound 4a was still obtained in low yields of $35 \%$ and $38 \%$ respectively (Table 1 , 
entries 2 and 3). Next we used $\mathrm{MeCN}$ in the presence of various $\mathrm{Cu}(\mathrm{I})$ species; $\mathrm{CuBr}$ and $\mathrm{CuI}$ were found to improve the yields of $\mathbf{4 a}$ to $65 \%$ and $57 \%$, respectively (Table 1, entries 4 and 5). When molecular sieves (MS) were added to the reaction mixture, 4a was obtained in $85 \%$ yield (Table 1, entry 6). Further examination of solvent effects revealed that THF, in the presence of molecular sieves, afforded a result similar to that noted when using $\mathrm{MeCN}$ as solvent (Table 1, entry 7). Very low yield was obtained when using $\mathrm{CH}_{2} \mathrm{Cl}_{2}$ or $\mathrm{PhMe}$ as solvents (Table 1, entries 8 and 9). Consequently, the optimal reaction conditions were assigned to include 4.0 equivalents of zinc and 1.5 equivalents of $\mathrm{CuBr}$ in the presence of molecular sieves in $\mathrm{MeCN}$ at $60{ }^{\circ} \mathrm{C}$ for $3 \mathrm{~h}$.

Using the optimized conditions, the scope and generality of the method was examined. A number of benzotriazolated nitrones $\mathbf{2} \mathbf{a}-\mathbf{h}$ and 2-bromonitriles with aryl and heteroaryl groups were converted to the corresponding trisubstituted 5-imino-1,2-oxazoles 4a-i in good to high yields (75-91\%) in short time under mild conditions (Scheme 3, Table 2).

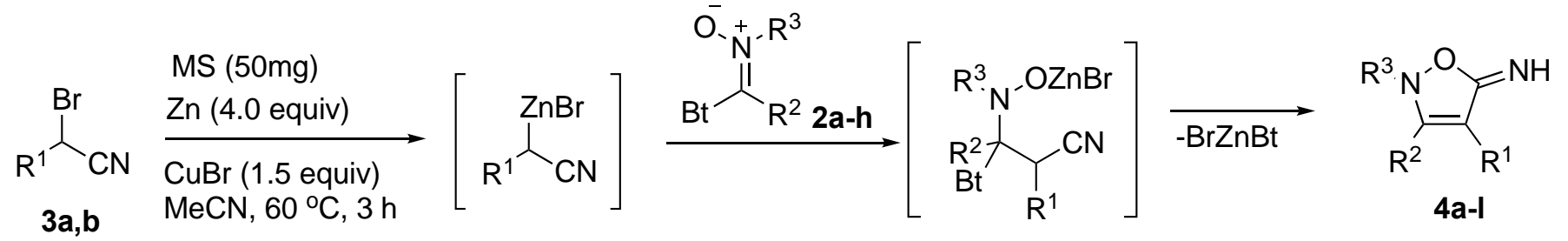

Scheme 3. Reformatsky reaction of 1-bromonitriles 3a,b and $C$-benzotriazolated nitrones $\mathbf{2 a - h}$

Table 2. The yields of trisubstituted 5-imino-1,2-oxazoles 4a-l

\begin{tabular}{ccccc}
\hline Product & $\mathrm{R}^{1}$ & $\mathrm{R}^{2}$ & $\mathrm{R}^{3}$ & Yield, \% \\
\hline $\mathbf{4 a}$ & $\mathrm{Me}$ & $4-\mathrm{MeC}_{6} \mathrm{H}_{4}$ & $4-\mathrm{MeC}_{6} \mathrm{H}_{4}$ & 85 \\
$\mathbf{4 b}$ & $\mathrm{Me}$ & $\mathrm{Ph}$ & $\mathrm{Ph}$ & 98 \\
$\mathbf{4 c}$ & $\mathrm{Me}$ & furan-2-yl & $\mathrm{Ph}$ & 89 \\
$\mathbf{4 d}$ & $\mathrm{Me}$ & thiophen-2-yl & $\mathrm{Ph}$ & 82 \\
$\mathbf{4 e}$ & $\mathrm{Me}$ & pyridin-3-yl & $\mathrm{Ph}$ & 84 \\
$\mathbf{4 f}$ & $\mathrm{Me}$ & $4-\mathrm{MeOC}_{6} \mathrm{H}_{4}$ & $\mathrm{Ph}$ & 75 \\
$\mathbf{4 g}$ & $\mathrm{Me}$ & $4-\mathrm{MeOC}_{6} \mathrm{H}_{4}$ & $4-\mathrm{ClC}_{6} \mathrm{H}_{4}$ & 79 \\
$\mathbf{4 h}$ & $\mathrm{Me}$ & $4-\mathrm{MeOC}_{6} \mathrm{H}_{4}$ & $4-\mathrm{MeOC}_{6} \mathrm{H}_{4}$ & 78 \\
$\mathbf{4 i}$ & $\mathrm{Ph}$ & $4-\mathrm{MeC}_{6} \mathrm{H}_{4}$ & $4-\mathrm{MeC}_{6} \mathrm{H}_{4}$ & 82 \\
$\mathbf{4 j}$ & $\mathrm{Ph}$ & furan-2-yl & $\mathrm{Ph}$ & 80 \\
$\mathbf{4 k}$ & $\mathrm{Ph}$ & thiophen-2-yl & $\mathrm{Ph}$ & 75 \\
$\mathbf{4 l}$ & $\mathrm{Ph}$ & pyridin-3-yl & $\mathrm{Ph}$ & \\
\hline
\end{tabular}


Next, we investigated the synthetic potential of 5-imino-1,2-oxazoles 4a,c,e as precursors in the preparation of 5-functionalized 1,2-oxazole derivatives. Reactions of 5-imino-1,2-oxazoles 4a,c,e with phenyl isocyanate afforded the corresponding urea derivatives 5a-c in high yields (85-92\%). Acidic hydrolysis of 5-imino-1,2-oxazole $\mathbf{4 a}$ with $20 \% \mathrm{HCl}$ afforded the 1,2-oxazol-5-one $\mathbf{6 a}$ in high yield (95\%) (Scheme 4, Table 3).

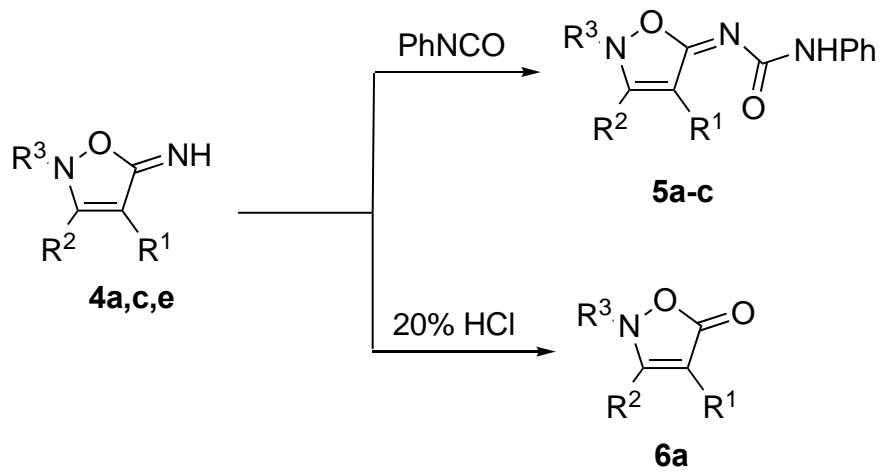

Scheme 4. Reactions of 5-imino-1,2-oxazoles 4a,c,e with phenyl isocyanates and $20 \% \mathrm{HCl}$

Table 3. The yields of 5-functionalized 1,2-oxazoles

\begin{tabular}{cccccc}
\hline Starting material & Product & $\mathrm{R}^{1}$ & $\mathrm{R}^{2}$ & $\mathrm{R}^{3}$ & Yield, \% \\
\hline $\mathbf{4 a}$ & $\mathbf{5 a}$ & $\mathrm{Me}$ & $4-\mathrm{MeC}_{6} \mathrm{H}_{4}$ & $4-\mathrm{MeC}_{6} \mathrm{H}_{4}$ & 92 \\
$\mathbf{4 c}$ & $\mathbf{5 b}$ & $\mathrm{Me}$ & furan-2-yl & $\mathrm{Ph}$ & 88 \\
$\mathbf{4 e}$ & $\mathbf{5 c}$ & $\mathrm{Me}$ & pyridin-3-yl & $\mathrm{Ph}$ & 85 \\
$\mathbf{4 a}$ & $\mathbf{6 a}$ & $\mathrm{Me}$ & $4-\mathrm{MeC}_{6} \mathrm{H}_{4}$ & $4-\mathrm{MeC}_{6} \mathrm{H}_{4}$ & 95 \\
\hline
\end{tabular}

The smooth conversion of 5-imino-1,2-oxazole 4a into 1,2-oxazol-5-one 6a promoted us to develop the one-pot protocol for the synthesis of 1,2-oxazol-5-ones 6a-c. Treatment of 2-bromopropionitrile (3a) with compounds 2a,c,d in THF afforded 5-imino-1,2-oxazoles 4a,c,d as intermediates, which upon treatment with $20 \% \mathrm{HCl}$ at room temperature for $3 \mathrm{~h}$ underwent hydrolysis to give 1,2-oxazol-5-ones 6a-c in high yields (85-90\%) (Scheme 5, Table 4). The overall yield of 1,2-oxazol-5-one 6a is comparable in one-pot and multistep procedures.

Table 4. One-pot synthesis of 1,2-oxazol-5-ones 6

\begin{tabular}{ccccc}
\hline Product & $\mathrm{R}^{1}$ & $\mathrm{R}^{2}$ & $\mathrm{R}^{3}$ & Yield, \% \\
\hline 6a & $\mathrm{Me}$ & $4-\mathrm{MeC}_{6} \mathrm{H}_{4}$ & $4-\mathrm{MeC}_{6} \mathrm{H}_{4}$ & 85 \\
$\mathbf{6 b}$ & $\mathrm{Me}$ & thiophen-2-yl & $\mathrm{Ph}$ & 88 \\
$\mathbf{6 c}$ & $\mathrm{Me}$ & furan-2-yl & $\mathrm{Ph}$ & 90
\end{tabular}


The structures of all synthesized compounds (4-6) were all elucidated by $\mathrm{IR}{ }^{1} \mathrm{H},{ }^{13} \mathrm{C}$ NMR, MS spectroscopy and elemental analysis.

In conclusion, we have synthesized a variety of novel 5-imino-1,2-oxazole derivatives, furthermore, we have developed a new and operationally simple one-pot protocol for the synthesis of 1,2-oxazol-5-ones.

\section{EXPERIMENTAL}

Melting points were determined on a Reichert hot-stage microscope and are uncorrected; The NMR spectra were recorded eith a Varian $300 \mathrm{MHz}$ instrument $\left(300 \mathrm{MHz}\right.$ for ${ }^{1} \mathrm{H}$ and $75 \mathrm{MHz}$ for ${ }^{13} \mathrm{C}$ ) in DMSO- $d_{6}$ using TMS as an internal standard. IR spectra were measured on Shimadzu FTIR-8300 spectrophotometer. MS spectra were measured by a JEOL JMS AX505 HA spectrometer. Column chromatography was performed on silica gel 200-245 mesh. C-Benzotriazolated nitrones 2a-h were prepared according to previously published procedures. ${ }^{16}$

Synthesis of 5-imino-1,2-oxazoles 4a-l (general method): 1-Bromonitrile 3a,b (1.0 mmol) and molecular sieves ( $4 \AA$, $50 \mathrm{mg})$ were added to a suspension of zinc $(4.0 \mathrm{mmol})$ and $\mathrm{CuBr}(1.5 \mathrm{mmol}) \mathrm{in}$ $\mathrm{MeCN}(3.0 \mathrm{~mL})$ and the mixture was stirred for $1 \mathrm{~h}$ at room temperature followed by addition of $C$-benzotriazolated nitrones $\mathbf{2 a - h}(1.0 \mathrm{mmol})$ dissolved in $\mathrm{MeCN}(1 \mathrm{~mL})$. The reaction mixture was heated to $60{ }^{\circ} \mathrm{C}$ for $3 \mathrm{~h}$ and cooled to room temperature. The mixture was filtered, concentrated under reduced pressure and the residue subjected to column chromatography on silica gel using EtOAc-hexane, 9:1 as eluent.

4-Methyl-2,3-di(p-tolyl)isoxazol-5(2H)-imine (4a): Yield $237 \mathrm{mg}$ (85\%), colorless crystals, mp 192-194 ${ }^{\circ} \mathrm{C}$; IR (KBr): v $1660 \mathrm{~cm}^{-1}(\mathrm{C}=\mathrm{N}) .{ }^{1} \mathrm{H}$ NMR $\delta 2.02(\mathrm{~s}, 3 \mathrm{H}), 2.22(\mathrm{~s}, 3 \mathrm{H}), 2.30(\mathrm{~s}, 3 \mathrm{H}), 6.93(\mathrm{~d}, J=8.1 \mathrm{~Hz}$, $2 \mathrm{H}), 7.23(\mathrm{~d}, J=8.0 \mathrm{~Hz}, 2 \mathrm{H}), 7.33(\mathrm{~d}, J=8.1 \mathrm{~Hz}, 2 \mathrm{H}), 7.84(\mathrm{~d}, J=8.0 \mathrm{~Hz}, 2 \mathrm{H}) ;{ }^{13} \mathrm{C}$ NMR $\delta 20.7,21.3$, 21.4, 118.3, 128.1, 128.7, 129.1, 129.3, 132.0, 132.6, 137.4, 139.8, 147.4, 165.1; MS m/z: 278. Anal. Calcd. for $\mathrm{C}_{18} \mathrm{H}_{18} \mathrm{~N}_{2} \mathrm{O}$ : C, 77.67; H, 6.52; N, 10.06. Found: C, 77.52; H, 6.40; N, 10.10.

4-Methyl-2,3-diphenylisoxazol-5(2H)-imine (4b): Yield $220 \mathrm{mg}(88 \%)$, colorless crystals, mp 186-188 ${ }^{\circ} \mathrm{C}$; IR (KBr): v $1665 \mathrm{~cm}^{-1}(\mathrm{C}=\mathrm{N}) .{ }^{1} \mathrm{H}$ NMR $\delta 1.93(\mathrm{~s}, 3 \mathrm{H}), 6.66(\mathrm{~d}, J=8.1 \mathrm{~Hz}, 2), 7.26-7.46(\mathrm{~m}, 6 \mathrm{H})$, 7.58-7.64 (m, 2H); ${ }^{13} \mathrm{C}$ NMR $\delta 18.0,116.6,124.8,126.7,128.6,128.7,128.9,129.3,131.8,137.4,147.3$, 162.2; MS m/z: 250. Anal. Calcd. for $\mathrm{C}_{16} \mathrm{H}_{14} \mathrm{~N}_{2} \mathrm{O}: \mathrm{C}, 76.78 ; \mathrm{H}, 5.64 ; \mathrm{N}, 11.19$. Found: C, 76.82; H, 5.72; N, 11.08.

3-Furan-2-yl-4-methyl-2-phenylisoxazol-5(2H)-imine (4c): Yield $219 \mathrm{mg}$ (91\%), colorless crystals, mp 162-164 ${ }^{\circ} \mathrm{C}$; IR (KBr): $v 1670 \mathrm{~cm}^{-1}(\mathrm{C}=\mathrm{N}) .{ }^{1} \mathrm{H}$ NMR $\delta 1.89(\mathrm{~s}, 3 \mathrm{H}), 6.39(\mathrm{dd}, J=3.4,1.8 \mathrm{~Hz}, 1 \mathrm{H}), 6.67(\mathrm{~d}$, $J=8.1 \mathrm{~Hz}, 2 \mathrm{H}), 7.13(\mathrm{~d}, J=3.4,0.8 \mathrm{~Hz}, 1 \mathrm{H}), 7.35-7.63(\mathrm{~m}, 3 \mathrm{H}), 7.74(\mathrm{dd}, J=1.8,0.8 \mathrm{~Hz}, 1 \mathrm{H}) ;{ }^{13} \mathrm{C} \mathrm{NMR}$ $\delta 18.1,109.7,110.8,116.7,124.8,128.7,129.2$, 137.5, 143.1, 145.4, 147.4, 162.3; MS m/z: 240. Anal. Calcd. for $\mathrm{C}_{14} \mathrm{H}_{12} \mathrm{~N}_{2} \mathrm{O}_{2}$ : \%: C, 69.99; H, 5.03; N, 11.66. Found: C, 70.06; H, 5.10; N, 11.55. 
4-Methyl-2-phenyl-3-thiophen-2-ylisoxazol-5(2H)-imine (4d): Yield $228 \mathrm{mg}(89 \%)$, colorless crystals, mp 159-161 ${ }^{\circ} \mathrm{C}$; IR (KBr): $v 1666 \mathrm{~cm}^{-1}(\mathrm{C}=\mathrm{N}) .{ }^{1} \mathrm{H}$ NMR $\delta 1.94(\mathrm{~s}, 3 \mathrm{H}), 6.67(\mathrm{~d}, J=8.1 \mathrm{~Hz}, 2 \mathrm{H}), 7.13(\mathrm{dd}$, $J=7.1,4.5 \mathrm{~Hz}, 1 \mathrm{H}), 7.35(\mathrm{t}, J=7.5 \mathrm{~Hz}, 1 \mathrm{H}), 7.53(\mathrm{dd}, J=4.5,1.3 \mathrm{~Hz}, 1 \mathrm{H}), 7.60-7.68(3 \mathrm{H}, \mathrm{m}) ;{ }^{13} \mathrm{C} \mathrm{NMR}$ $\delta \quad 18.0,116.7,124.8,126.1,126.3,127.4,128.7,129.2,133.6,137.5,147.4,162.3$; MS m/z: 256. Anal. Cacld. for $\mathrm{C}_{14} \mathrm{H}_{12} \mathrm{~N}_{2} \mathrm{OS}$ : C, 65.60; H, 4.72; N, 10.93. Found: C, 65.51; H, 4.81; N, 11.01.

4-Methyl-2-phenyl-3-pyridin-3-ylisoxazol-5(2H)-imine (4e): Yield $206 \mathrm{mg}$ (82\%), colorless crystals, mp 201-203 ${ }^{\circ} \mathrm{C}$; IR (KBr): $v 1670 \mathrm{~cm}^{-1}(\mathrm{C}=\mathrm{N}) .{ }^{1} \mathrm{H}$ NMR $\delta 1.90(\mathrm{~s}, 3 \mathrm{H}), 6.67(\mathrm{~d}, J=8.1 \mathrm{~Hz}, 2 \mathrm{H}), 7.35(\mathrm{t}, J$ $=7.5 \mathrm{~Hz}, 1 \mathrm{H}), 7.46-7.75(\mathrm{~m}, 4 \mathrm{H}), 8.55(\mathrm{dd}, J=4.7,1.9 \mathrm{~Hz}, 1 \mathrm{H}), 8.92(\mathrm{dd}, J=1.9,1.6 \mathrm{~Hz}, 1 \mathrm{H}) .{ }^{13} \mathrm{C} \mathrm{NMR}$ $\delta 18.0,116.7,123.9,124.7,128.6,128.7,129.2,131.3,137.5,145.0,147.4,149.8,162.3$; MS m/z: 251. Anal. Calcd. for $\mathrm{C}_{15} \mathrm{H}_{13} \mathrm{~N}_{3} \mathrm{O}$ : C, 71.70; H, 5.21; N, 16.72. Found: C, 71.60; H, 5.11; N, 16.56 .

3-(p-Methoxyphenyl)-4-methyl-2-phenylisoxazol-5(2H)-imine (4f): Yield $235 \mathrm{mg}$ (84\%), colorless crystals, mp 182-184 ${ }^{\circ} \mathrm{C}$; IR (KBr): $v 1665 \mathrm{~cm}^{-1}(\mathrm{C}=\mathrm{N}) .{ }^{1} \mathrm{H}$ NMR $\delta 2.22(\mathrm{~s}, 3 \mathrm{H}), 3.73(\mathrm{~s}, 3 \mathrm{H}), 6.65(\mathrm{~d}, J=$ $8.1 \mathrm{~Hz}, 2 \mathrm{H}), 7.01(\mathrm{~d}, J=8.8 \mathrm{~Hz}, 2 \mathrm{H}), 7.35-7.53(\mathrm{~m}, 3 \mathrm{H}), 7.64(\mathrm{~d}, J=8.1 \mathrm{~Hz}, 2 \mathrm{H}) .{ }^{13} \mathrm{C}$ NMR $\delta 18.0,55.5$, 114.2, 116.7, 124.7, 128.7, 129.1, 129.2, 131.9, 137.5, 147.4, 160.4, 162.3; MS m/z: 280. Anal. Calcd. for $\mathrm{C}_{17} \mathrm{H}_{16} \mathrm{~N}_{2} \mathrm{O}_{2}$ : C, 72.84; H, 5.75; N, 9.99. Found: C, 72.68; H, 5.88; N, 10.07 .

2-(p-Chlorophenyl)-3-(p-methoxyphenyl)-4-methylisoxazol-5(2H)-imine (4g): Yield $236 \mathrm{mg}(75 \%)$, colorless crystals, mp: $210-212{ }^{\circ} \mathrm{C}$; IR (KBr): $v 1672 \mathrm{~cm}^{-1}(\mathrm{C}=\mathrm{N}) .{ }^{1} \mathrm{H}$ NMR $\delta 2.24(\mathrm{~s}, 3 \mathrm{H}), 3.77$ (s, 3H), $7.01(\mathrm{~d}, J=8.8 \mathrm{~Hz}, 2 \mathrm{H}), 7.50-7.60(\mathrm{~m}, 6 \mathrm{H}) .{ }^{13} \mathrm{C} \mathrm{NMR} \delta 18.0,55.5,114.2,124.9,127.8,129.1,129.2$, 129.4, 131.9, 137.5, 147.4, 160.4, 162.3; MS m/z: 314. Anal. Calcd. For $\mathrm{C}_{17} \mathrm{H}_{15} \mathrm{ClN}_{2} \mathrm{O}_{2}$ : C, 64.87; $\mathrm{H}$, 4.80; N, 8.90. Found: C, 64.92; H, 4.72; N, 8.73.

2,3-di-(p-Methoxyphenyl)-4-methylisoxazol-5(2H)-imine (4h): Yield $245 \mathrm{mg}$ (79\%), colorless crystals, mp 204-206 ${ }^{\circ} \mathrm{C}$; IR (KBr): v $1677 \mathrm{~cm}^{-1}(\mathrm{C}=\mathrm{N}) .{ }^{1} \mathrm{H}$ NMR $\delta 2.24(\mathrm{~s}, 3 \mathrm{H}), 3.75$ (s, 3H), 3.77 (s, 3H), $6.65(\mathrm{~d}$, $J=8.7 \mathrm{~Hz}, 2 \mathrm{H}), 6.82(\mathrm{~d}, J=8.7 \mathrm{~Hz}, 2 \mathrm{H}), 7.01(\mathrm{~d}, J=8.8 \mathrm{~Hz}, 2 \mathrm{H}), 7.53(\mathrm{~d}, J=8.8 \mathrm{~Hz}, 2 \mathrm{H}) ;{ }^{13} \mathrm{C}$ NMR $\delta$ 18.0, 55.4, 55.5, 114.2, 115.1, 117.3, 129.1, 129.3, 131.9, 137.5, 147.4, 156.9, 160.4, 162.3; MS m/z: 310. Anal. Calcd. for $\mathrm{C}_{18} \mathrm{H}_{18} \mathrm{~N}_{2} \mathrm{O}_{3}$ : C, 69.66; H, 5.85; N, 9.03. Found: C, 69.73; H, 5.93; N, 8.94.

4-Phenyl-2,3-di-(p-tolyl)isoxazol-5(2H)-imine (4i): Yield $266 \mathrm{mg}$ (78\%), colorless crystals, mp 198-200 ${ }^{\circ} \mathrm{C}$; IR $(\mathrm{KBr}): v 1660 \mathrm{~cm}^{-1}(\mathrm{C}=\mathrm{N}) .{ }^{1} \mathrm{H}$ NMR $\delta 2.22(\mathrm{~s}, 3 \mathrm{H}), 2.31(\mathrm{~s}, 3 \mathrm{H}), 7.03(\mathrm{~d}, J=8.1 \mathrm{~Hz}, 2 \mathrm{H})$, 7.26-7.38 (m, $7 \mathrm{H}), 7.46(\mathrm{~d}, J=8.8 \mathrm{~Hz}, 2 \mathrm{H}), 7.77(\mathrm{~d}, J=8.0 \mathrm{~Hz}, 2 \mathrm{H}) .{ }^{13} \mathrm{C}$ NMR $\delta 21.2,21.3,118.2,127.5$, 128.3, 128.6, 128.9, 129.0, 129.2, 129.3, 131.8, 132.4, 132.7, 137.5, 139.7, 147.4, 162.3; MS m/z: 340. Anal. Calcd. for $\mathrm{C}_{23} \mathrm{H}_{20} \mathrm{~N}_{2} \mathrm{O}$ : C, 81.15; H, 5.92; N, 8.23. Found: C, 81.34; H, 6.01; N, 8.12.

3-Furan-2-yl-2,4-diphenylisoxazol-5(2H)-imine (4j): Yield $248 \mathrm{mg}(82 \%)$, pale yellow crystals, $\mathrm{mp}$ 170-172 ${ }^{\circ} \mathrm{C}$; IR (KBr): $v 1672 \mathrm{~cm}^{-1}(\mathrm{C}=\mathrm{N}) .{ }^{1} \mathrm{H}$ NMR $\delta 6.56(\mathrm{dd}, J=3.5,1.8 \mathrm{~Hz}, 1 \mathrm{H}), 7.06(\mathrm{~d}, J=8.2 \mathrm{~Hz}$, $2 \mathrm{H}), 7.24-7.44(\mathrm{~m}, 7 \mathrm{H}), 7.65(\mathrm{~d}, J=8.2 \mathrm{~Hz}, 2 \mathrm{H}), 7.81(\mathrm{dd}, J=1.8,0.8 \mathrm{~Hz}, 1 \mathrm{H}) ;{ }^{13} \mathrm{C}$ NMR $\delta 109.7,110.8$, 
116.7, 124.7, 127.5, 128.6, 128.7, 128.9, 129.2, 132.7, 137.5, 143.0, 145.4, 147.4, 162.3; MS m/z: 302. Anal. Calcd. for $\mathrm{C}_{19} \mathrm{H}_{14} \mathrm{~N}_{2} \mathrm{O}_{2}$ : C, 75.48; H, 4.67; N, 9.27. Found: C, 75.56; H, 4.78; N, 9.14.

2,4-Diphenyl-3-thiophen-2-ylisoxazol-5(2H)-imine (4k): Yield $255 \mathrm{mg}(80 \%)$, pale yellow crystals, $\mathrm{mp}$ 168-170 ${ }^{\circ} \mathrm{C}$; IR (KBr): $v 1665 \mathrm{~cm}^{-1}(\mathrm{C}=\mathrm{N}) .{ }^{1} \mathrm{H}$ NMR $\delta 7.05(\mathrm{~d}, J=8.2 \mathrm{~Hz}, 2 \mathrm{H}), 7.19(\mathrm{dd}, J=7.3,5.2 \mathrm{~Hz}$, $1 \mathrm{H}), 7.27-7.31(\mathrm{~m}, 2 \mathrm{H}), 7.42-7.55(\mathrm{~m}, 5 \mathrm{H}), 7.65(\mathrm{~d}, J=8.2 \mathrm{~Hz}, 2 \mathrm{H}), 7.78(\mathrm{dd}, J=7.3,1.3 \mathrm{~Hz}, 1 \mathrm{H}) ;{ }^{13} \mathrm{C}$ NMR $\delta 116.7,124.7,126.1,126.3,127.4,127.5,128.6,128.7,128.9,129.2,132.7,133.6,137.5,147.4$, 162.3; MS m/z: 318. Anal. Calcd. for $\mathrm{C}_{19} \mathrm{H}_{14} \mathrm{~N}_{2} \mathrm{OS}$ : C, 71.67; $\mathrm{H}, 4.43 ; \mathrm{N}, 8.80$. Found: C, 71.56; H, 4.51; $\mathrm{N}, 8.95$.

2,4-Diphenyl-3-pyridin-3-ylisoxazol-5(2H)-imine (4l): Yield $235 \mathrm{mg}$ (75\%), colorless crystals, $\mathrm{mp}$ 218-220 ${ }^{\circ} \mathrm{C}$; IR (KBr): $v 1666 \mathrm{~cm}^{-1}(\mathrm{C}=\mathrm{N}) .{ }^{1} \mathrm{H}$ NMR $\delta 7.05(\mathrm{~d}, J=8.2 \mathrm{~Hz}, 2 \mathrm{H}), 7.27-7.52(\mathrm{~m}, 7 \mathrm{H}), 7.65$ (d, $J=8.2 \mathrm{~Hz}, 2 \mathrm{H}), 7.99$ (ddd, $J=8.2,1.9,1.7 \mathrm{~Hz}, 1 \mathrm{H}), 8.59$ (ddd, $J=4.8,1.9,1.9 \mathrm{~Hz}, 1 \mathrm{H}), 8.93$ (ddd, $J$ $=1.9,1.7,0.5 \mathrm{~Hz}, 1 \mathrm{H}) ;{ }^{13} \mathrm{C} \mathrm{NMR} \delta 116.7,123.9,124.7,127.5,128.5,128.6,128.7,128.9,129.2,131.3$, 132.7, 137.5, 145.0, 147.4, 149.7, 162.3; MS m/z: 313. Anal. Calcd. for $\mathrm{C}_{20} \mathrm{H}_{15} \mathrm{~N}_{3} \mathrm{O}: \mathrm{C}, 76.66 ; \mathrm{H}, 4.82 ; \mathrm{N}$, 13.41. Found: C, 76.82; H, 4.90; N, 13.29.

Synthesis of compounds 5a-c (general method): Phenyl isocyanate $(1.0 \mathrm{mmol})$ was added to a solution of 4a-c $(1.0 \mathrm{mmol})$ in anhydrous THF $(5.0 \mathrm{~mL})$ under ice cooling, and the reaction mixture was stirred at room temperature for $1 \mathrm{~h}$. The solvent was evaporated under reduced pressure and the remaining mixture was purified by column chromatography on silica gel with EtOAc-hexane, 8:2 as an eluent. Crystallization from EtOAc-hexane afforded compounds 5a-c as white powder.

1-(4-Methyl-2,3-di(p-tolyl)isoxazol-5(2H)-ylidene)-3-phenylurea (5a): Yield $366 \mathrm{mg}$ (92\%), colorless crystals, mp 207-209 ${ }^{\circ} \mathrm{C}$; IR (KBr): v 1695, $1720 \mathrm{~cm}^{-1}(\mathrm{C}=\mathrm{N}, \mathrm{C}=\mathrm{O}) .{ }^{1} \mathrm{H}$ NMR $\delta 2.22(\mathrm{~s}, 3 \mathrm{H}), 2.25$ (s, 3H), $2.34(\mathrm{~s}, 3 \mathrm{H}), 7.08(\mathrm{t}, J=7.8 \mathrm{~Hz}, 1 \mathrm{H}), 7.21-7.37(\mathrm{~m}, 8 \mathrm{H}), 7.45(\mathrm{~d}, J=8.1 \mathrm{~Hz}, 2 \mathrm{H}), 7.96(\mathrm{~d}, J=8.0 \mathrm{~Hz}$, $2 \mathrm{H}) ;{ }^{13} \mathrm{C}$ NMR $\delta 18.0,21.2,21.3,118.2,118.9,124.7,128.3,128.9,129.1,129.2,129.3,131.8,132.4$, 135.5, 137.5, 139.7, 147.4, 158.2, 162.3; MS m/z: 397. Anal. Calcd. for $\mathrm{C}_{25} \mathrm{H}_{23} \mathrm{~N}_{3} \mathrm{O}_{2}$ : C, 75.55; H, 5.83; N, 10.57. Found: C, 75.61; H, 5.72; N, 10.69.

1-(3-Furan-2-yl-4-Methyl-2-phenylisoxazol-5(2H)-ylidene)-3-phenylurea (5b): Yield 316 mg (88\%), colorless crystals, mp 201-203 ${ }^{\circ} \mathrm{C}$; IR (KBr): $v$ 1690, $1725 \mathrm{~cm}^{-1}(\mathrm{C}=\mathrm{N}, \mathrm{C}=\mathrm{O}) .{ }^{1} \mathrm{H}$ NMR $\delta 2.24$ (s, 3H), $6.57(\mathrm{dd}, J=3.5,1.8 \mathrm{~Hz}, 1 \mathrm{H}), 7.08(\mathrm{t}, J=7.8 \mathrm{~Hz}, 1 \mathrm{H}), 7.27-7.44(\mathrm{~m}, 6 \mathrm{H}), 7.63-7.77$ (m, 4H), 7.90 (dd, $J$ $=1.8,0.8 \mathrm{~Hz}, 1 \mathrm{H}) ;{ }^{13} \mathrm{C}$ NMR $\delta 18.0,109.7,110.8,116.7,118.9,124.7,125.0,128.7,129.1,129.2,135.5$, 137.5, 143.0, 145.4, 147.4, 158.2, 162.3; MS m/z: 359. Anal. Calcd. for $\mathrm{C}_{21} \mathrm{H}_{17} \mathrm{~N}_{3} \mathrm{O}_{3}: \mathrm{C}, 70.18 ; \mathrm{H}, 4.77 ; \mathrm{N}$, 11.69. Found: C, 70.01; H, 4.59; N, 11.78.

1-(4-Methyl-2-phenyl-3-pyridin-3-ylisoxazol-5(2H)-ylidene)-3-phenylurea (5c): Yield $315 \mathrm{mg}(85 \%)$, colorless crystals, mp 240-242 ${ }^{\circ} \mathrm{C}$; IR (KBr): $v$ 1695, $1722 \mathrm{~cm}^{-1}(\mathrm{C}=\mathrm{N}, \mathrm{C}=\mathrm{O}) .{ }^{1} \mathrm{H}$ NMR $\delta 2.22(\mathrm{~s}, 3 \mathrm{H})$, $7.08(\mathrm{t}, J=7.8 \mathrm{~Hz}, 1 \mathrm{H}), 7.27-7.50(\mathrm{~m}, 6 \mathrm{H}), 7.60-7.70(\mathrm{~m}, 4 \mathrm{H}), 7.98(\mathrm{ddd}, J=8.2,1.9,1.6 \mathrm{~Hz}, 1 \mathrm{H}), 8.65$ 
$(\mathrm{ddd}, J=4.7,1.9,1.8 \mathrm{~Hz}, 1 \mathrm{H}), 9.19(\mathrm{ddd}, J=1.9,1.6,0.5 \mathrm{~Hz}, 1 \mathrm{H}) ;{ }^{13} \mathrm{C}$ NMR $\delta 18.0,116.7,118.9,123.9$, 124.7, 125.0, 128.6, 128.7, 129.1, 129.2, 131.3, 135.5, 137.5, 145.0, 147.4, 149.8, 158.2, 162.3; MS m/z: 370. Anal. Calcd. for $\mathrm{C}_{22} \mathrm{H}_{18} \mathrm{~N}_{4} \mathrm{O}_{2}$ : C, 71.34; H, 4.90; N, 15.13. Found: C, 71.22; H, 5.03; N, 14.96 .

Synthesis of 1,2-oxazol-5-one 6a from 4-methyl-5-phenylcarbamoylimino-2,3-di( $p$-tolyl)-2H-1,2oxazole (6a):

Aqueous $\mathrm{HCl}(5.0 \mathrm{~mL}, 20 \%)$ was added to a solution of $4(1.0 \mathrm{mmol})$ in THF $(3.0 \mathrm{~mL})$ and the mixture was stirred at room temperature for $3 \mathrm{~h}$. The mixture was extracted with $\mathrm{CH}_{2} \mathrm{Cl}_{2}(3 \times 5.0 \mathrm{~mL})$, the combined extracts were dried over $\mathrm{MgSO}_{4}$ and the solvent was evaporated. The resulting residue was chromatographed using EtOAc-hexane, 8:2.

\section{One-pot synthesis of 1,2-oxazol-5-ones 6a-c (general method):}

1-Bromonitrile $(1.0 \mathrm{mmol})$ and molecular sieves $(4 \AA$, $50 \mathrm{mg})$ were added to a suspension of zinc (1.0 $\mathrm{mmol})$ and $\mathrm{CuBr}(0.25 \mathrm{mmol})$ in THF $(3.0 \mathrm{~mL})$ and the mixture was stirred for $1 \mathrm{~h}$ at room temperature followed by addition of $2(1.0 \mathrm{mmol})$ dissolved in THF $(1.0 \mathrm{~mL})$. The reaction mixture was heated to 60 ${ }^{\circ} \mathrm{C}$ for $3 \mathrm{~h}$ and cooled to room temperature. Aqueous $\mathrm{HCl}(5.0 \mathrm{~mL}, 20 \%)$ was added to the mixture and stirred at room temperature for $3 \mathrm{~h}$. The mixture was extracted with $\mathrm{CH}_{2} \mathrm{Cl}_{2}(3 \times 7.0 \mathrm{~mL})$, the combined extracts were dried over $\mathrm{MgSO}_{4}$ and the solvent was evaporated. The resulting residue was chromatographed using EtOAc-hexane 8:2.

4-Methyl-2,3-di-(p-tolyl)isoxazol-5(2H)-one (6a): Yield $224 \mathrm{mg}$ (85\%), colorless crystals, mp 172-174 ${ }^{\circ} \mathrm{C}$; IR (KBr): $v 1730 \mathrm{~cm}^{-1}(\mathrm{C}=\mathrm{O}) .{ }^{1} \mathrm{H}$ NMR $\delta 1.91(\mathrm{~s}, 3 \mathrm{H}), 2.22(\mathrm{~s}, 3 \mathrm{H}), 2.29(\mathrm{~s}, 3 \mathrm{H}), 6.99(\mathrm{~d}, J=8.1 \mathrm{~Hz}$, $2 \mathrm{H}), 7.13(\mathrm{~d}, J=8.0 \mathrm{~Hz}, 2 \mathrm{H}), 7.34(\mathrm{~d}, J=8.1 \mathrm{~Hz}, 2 \mathrm{H}), 7.77(\mathrm{~d}, J=8.0 \mathrm{~Hz}, 2 \mathrm{H}) .{ }^{13} \mathrm{C}$ NMR $\delta 18.0,21.2$, 21.3, 118.2, 128.3, 128.9, 129.2, 129.3, 131.8, 132.5, 137.5, 139.8, 147.4, 169.1; MS m/z: 263. Anal. Calcd. for $\mathrm{C}_{18} \mathrm{H}_{17} \mathrm{NO}$ : C, 77.40; H, 6.13; N, 5.01. Found: C, 77.53; H, 6.27; N, 4.92.

4-Methyl-2-phenyl-3-thiophen-2-ylisoxazol-5(2H)-one (6b): Yield $226 \mathrm{mg}(88 \%)$, colorless crystals, mp 142-144 ${ }^{\circ} \mathrm{C}$; IR (KBr): $v 1735 \mathrm{~cm}^{-1}(\mathrm{C}=\mathrm{O}) .{ }^{1} \mathrm{H}$ NMR $\delta 1.91(\mathrm{~s}, 3 \mathrm{H}), 6.95(\mathrm{~m}, 2 \mathrm{H}), 7.13(\mathrm{dd}, J=7.0$, $4.5 \mathrm{~Hz}, 1 \mathrm{H}), 7.31(\mathrm{t}, J=7.5 \mathrm{~Hz}, 1 \mathrm{H}), 7.64-7.70(\mathrm{~m}, 3 \mathrm{H}), 7.75(\mathrm{dd}, J=7.0,1.3 \mathrm{~Hz}, 1 \mathrm{H}) ;{ }^{13} \mathrm{C} \mathrm{NMR} \delta 18.0$, 116.7, 124.7, 126.1, 126.3, 127.4, 128.7, 129.2, 133.6, 137.5, 147.4, 169.1; MS m/z: 257. Anal. Calcd. for $\mathrm{C}_{14} \mathrm{H}_{11} \mathrm{NO}_{2} \mathrm{~S}: \mathrm{C}, 65.35 ; \mathrm{H}, 4.31 ; \mathrm{N}$, 5.44. Found: C, 65.46; H, 4.44; N, 5.29.

3-Furan-2-yl-4-methyl-2-phenylisoxazol-5(2H)-one (6c): Yield $217 \mathrm{mg}$ (90\%), colorless crystals, mp 138-140 ${ }^{\circ} \mathrm{C}$; IR (KBr): $v 1730 \mathrm{~cm}^{-1}(\mathrm{C}=\mathrm{O}) .{ }^{1} \mathrm{H}$ NMR $\delta 1.90(\mathrm{~s}, 3 \mathrm{H}), 6.55(\mathrm{dd}, J=3.5,1.8 \mathrm{~Hz}, 1 \mathrm{H}), 7.25$ $(\mathrm{dd}, J=3.5,0.8 \mathrm{~Hz}, 1 \mathrm{H}), 7.28-7.64(\mathrm{~m}, 5 \mathrm{H}), 7.79(\mathrm{dd}, J=1.8,0.8 \mathrm{~Hz}, 1 \mathrm{H}) ;{ }^{13} \mathrm{C} \mathrm{NMR} \delta 18.0,109.7,110.8$, 116.7, 124.7, 128.7, 129.2, 137.5, 143.0, 145.4, 147.4, 169.1; MS $m / z: 241$. Anal. Calcd. for $\mathrm{C}_{14} \mathrm{H}_{11} \mathrm{NO}_{3}$ : C, 69.70; H, 4.60; N, 5.81. Found: C, 69.82; H, 4.48; N, 5.93. 


\section{REFERENCES}

1. M. Cox, M. Dixon, T. Lister, and R. H. P. Prager, Aust. J. Chem., 2004, 57, 455.

2. M. Cox, R. H. Prager, and D. M. R. Riessen, ARKIVOC, 2001, vii, 88.

3. A. D. Clark, U. T. Ha, R. H. Prager, and J. Smith, Aust. J. Chem., 1999, 52, 1029.

4. D. S. Millan and R. H. Prager, J. Chem. Soc., Perkin Trans. 1, 1998, 3245.

5. S. K. Laughlin, M. P. Clark, J. F. Djung, A. Golebiowski, T. A. Brugel, M. Sabat, R. G. Bookland, M. J. Laufersweiler, J. C. VanRens, J. A. Townes, B. De, L. C. Hsieh, S. C. Xu, R. L. Walter, M. J. Mekel, and M. J. Janusz, Bioorg. Med. Chem. Lett., 2005, 15, 2399.

6. F. Ikegami, A. Yamamoto, T. Sekine, T. Ishikawa, K. Kusamae-Eguch, T. Kusama, and K. Watanabe, Chem. Pharm. Bull., 2000, 48, 278.

7. S. A. Laufer and S. Margutti, J. Med. Chem., 2008, 51, 2580.

8. S. S. Mahajan, M. Scian, S. Sripathy, J. Posakony, U. Lao, T. K. Loe, V. Leko, A. Thalhofer, A. D. Schuler, A. Bedalov, and J. A. Simon, J. Med. Chem., 2014, 57, 3283.

9. Y. Tong, K. D. Stewart, S. Thomas, M. Przytulinska, E. F. Johnson, V. Klinghofer, J. Leverson, O. McCall, N. B. Soni, Y. Luo, N.-H. Lin, T. J. Sowin, V. L. Giranda, and T. D. Penning, Bioorg. Med. Chem. Lett., 2008, 18, 5206.

10. A. Minkkilä, J. R. Savinainen, H. Käsnänen, H. Xhaard, T. Nevalainen, J. T. Laitinen, A. Poso, J. Leppänen, and S. M. Saario, ChemMedChem, 2009, 4, 1253.

11. T. V. Hung, W. K. Janowski, and R. H. Prager, Aust. J. Chem., 1985, 38, 931.

12. A. R. Katritzky, K. Widyan, and K. Kirichenko, J. Org. Chem., 2007, 72, 5802; K. Widyan, Org. Prep. Proced. Int., 2021, 53, 120.

13. A. R. Katritzky, K. Widyan, and K. Gyanda, Synthesis, 2008, 1201; K. Widyan, Org. Prep. Proced. Int., 2021, 53, 455.

14. A. R. Katritzky, M. S. Kim, and K. Widyan, ARKIVOC, 2008, iii, 91.

15. K. Widyan, Eur. Chem. Bull., 2014, 3, 850.

16. P. Merino, S. Franco, F. L. Merchan, and T. Tejero, Tetrahedron:Asymmetry, 1997, 8, 3489.

17. R. F. Borch, M. D. Bernstein, and H. D. Durst, J. Am. Chem. Soc., 1971, 93, 2897.

18. D. Christenen and K. A. Jprgensen, J. Org. Chem., 1989, 54, 126. 